\title{
Analytical relations between kinetic-energy and particle densities for one-dimensional harmonically confined Fermi vapors
}

\author{
N. H. March \\ Department of Physics, University of Antwerp (RUCA), Antwerpen, Belgium \\ and Oxford University, Oxford, England \\ L. M. Nieto \\ Departamento de Física Teórica, Universidad de Valladolid, 47005 Valladolid, Spain
}

(Received 15 November 2000; published 16 March 2001)

\begin{abstract}
Analytical relations between kinetic-energy and particle densities are set out here, and used for numbers of harmonically confined independent fermions up to 100 particles. The use of positive definite kinetic-energy density is advocated in the present context, and this is then shown to tend to the von Weizsäcker inhomogeneity kinetic-energy density near the tunneling regime for the harmonic potential barrier, the example of 50 fermions being studied specifically.
\end{abstract}

DOI: 10.1103/PhysRevA.63.044502

PACS number(s): 31.15.Ew, 05.30.Fk, 71.10.Ca, 03.75.Fi

In a recent study, Vignolo, Minguzzi, and Tosi [1] presented numerical results for the particle density $n(x)$ and the kinetic pressure $P(x)$ obtained by a numerically converged procedure invoking a Green's function operator, up to $N$ $=1000$ fermions. As they pointed out, alternative methods exist for small $N$ in terms of Hermite polynomials $[2,3]$ and in particular for $N=5$ the kinetic-energy density $t(x)$ was analyzed also by Holas and March [4]. These workers displayed numerically the fact that for this small value of $N$ the von Weizsäcker inhomogeneity kinetic energy $t_{W}$ defined by

$$
t_{W}=\frac{1}{8} \frac{n^{\prime}(x)^{2}}{n(x)}
$$

already accounted for the tail of the kinetic energy density [see also Eq. (9) below].

Here we shall supply analytical relations between $n(x)$ and $t(x)$ for arbitrary $N$ to complement the numerical study of [1]. Starting with $t(x)$, we quote first the result of Lawes and March [3], namely, their Eq. (4.3),

$$
\begin{aligned}
t(x)= & \frac{1}{12} \psi_{N}^{2}\left[x^{4}-x^{2}(4 N+1)+2 N+4 N^{2}\right] \\
& -\frac{1}{12} \psi_{N}^{\prime 2}\left[x^{2}+1-2 N\right]+\frac{1}{6} x \psi_{N} \psi_{N}^{\prime},
\end{aligned}
$$

where $\psi_{N}$ is the normalized wave function for the $N$ th state, the lowest level corresponding to the case $N=1$.

The exact analytical result corresponding to Eq. (2) for the particle density is

$$
n(x)=\frac{1}{2} \psi_{N}^{\prime 2}-\frac{1}{2}\left(x^{2}-2 N\right) \psi_{N}^{2}
$$

Multiplying Eq. (3) throughout by $\frac{1}{6}\left(x^{2}-2 N\right)$ and adding the result to Eq. (2) readily yields

$$
\begin{aligned}
t(x)+\frac{1}{6}\left(x^{2}-2 N\right) n(x) & \\
& =\frac{1}{6} x \psi_{N} \psi_{N}^{\prime}-\frac{1}{12} \psi_{N}^{\prime 2}+\frac{1}{12}\left(2 N-x^{2}\right) \psi_{N}^{2} .
\end{aligned}
$$

Vignolo et al. [1] argue that asymptotically for $N$ large

$$
\frac{P_{L D A}(x)}{2}=t_{N \text { large }}(x)=\frac{1}{6 \pi}\left(2 N-x^{2}\right)^{3 / 2}
$$

and

$$
n_{L D A}(x)=n_{N \text { large }}(x)=\frac{1}{\pi}\left(2 N-x^{2}\right)^{1 / 2} .
$$

Inserting these results into the left-hand side (LHS) of Eq. (4) gives zero. This can be readily understood by using the result of Lawes and March [3] that

$$
n^{\prime}(x)=-x \psi_{N}^{2}+\psi_{N} \psi_{N}^{\prime},
$$

and forming $n^{\prime \prime}(x)$ from Eq. (7) allows Eq. (4) to be rewritten, after a short calculation, as

$$
t(x)+\frac{1}{6}\left(x^{2}-2 N\right) n(x)=-\frac{n^{\prime \prime}(x)}{12} .
$$

Thus, putting the LHS of Eq. (8) to zero is equivalent to neglecting the second derivative $n^{\prime \prime}(x)$, which is indeed consistent with the philosophy of the Thomas-Fermi approximation reflected in Eqs. (5) and (6). As Vignolo et al. [1] emphasize, $t(x)$ in Eq. (8) can become negative for $x^{2} \geq 2 N$.

To avoid this circumstance, we shall introduce the alternative definition of kinetic-energy density by using the $(\operatorname{grad} \psi)^{2}$ wave function form, which we shall define as $t_{\text {gradient }}(x) \equiv t_{G}(x)$. This differs from $t(x)$ in Eq. (8) by 

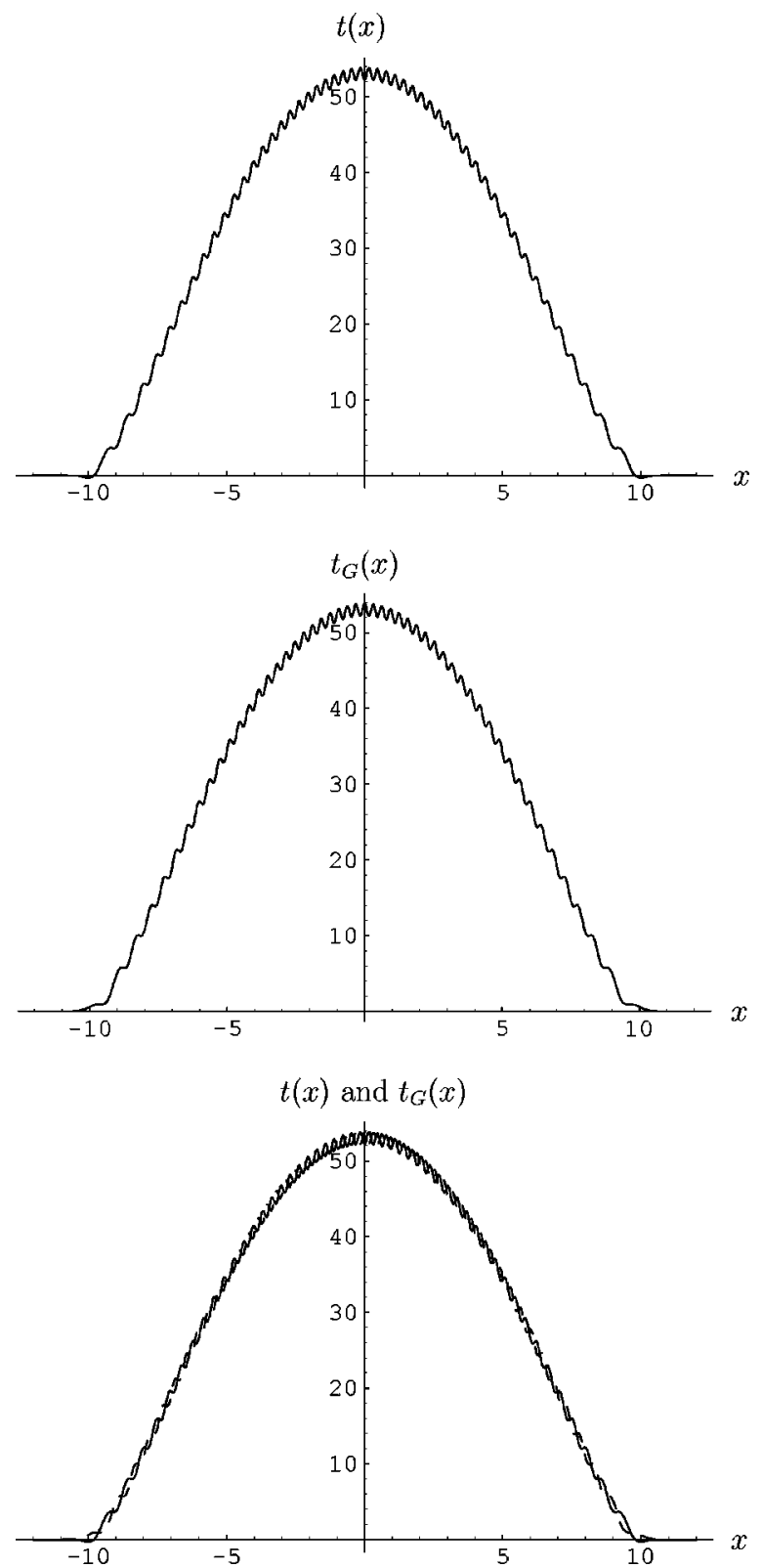

FIG. 1. Alternative forms $t$ and $t_{G}$ of kinetic-energy density are shown in the upper and middle parts of the figure for the case $N$ $=50$. The "antiphase" behavior of $t$ and $t_{G}$ is illustrated by their juxtaposition in the lowest part of the figure. Note that $t_{G}(x)$ defined in Eq. (9) is everywhere greater than zero, whereas $t(x)$ has a negative region as stressed by Vignolo et al. [1].

$$
t_{G}(x)=t(x)+\frac{n^{\prime \prime}(x)}{4} \text {. }
$$

For $N=50$, both $t_{G}(x)$ and $t(x)$ are plotted in Fig. 1 by means of Eqs. (3) and (8), and as anticipated above the small negative region in $t(x)$ is avoided in $t_{G}(x)$. Also, $t(x)$ has a subsidiary minimum at the origin, whereas $t_{G}(x)$ has a maximum, the antiphase behavior of $t(x)$ and $t_{G}(x)$ being made plain in the lowest part of Fig. 1 . The quantity $2 t_{G}(x)$ affords a more natural definition of the kinetic pressure than $2 t(x)$, because $2 t_{G}(x)$ is positive definite, as already stressed above.
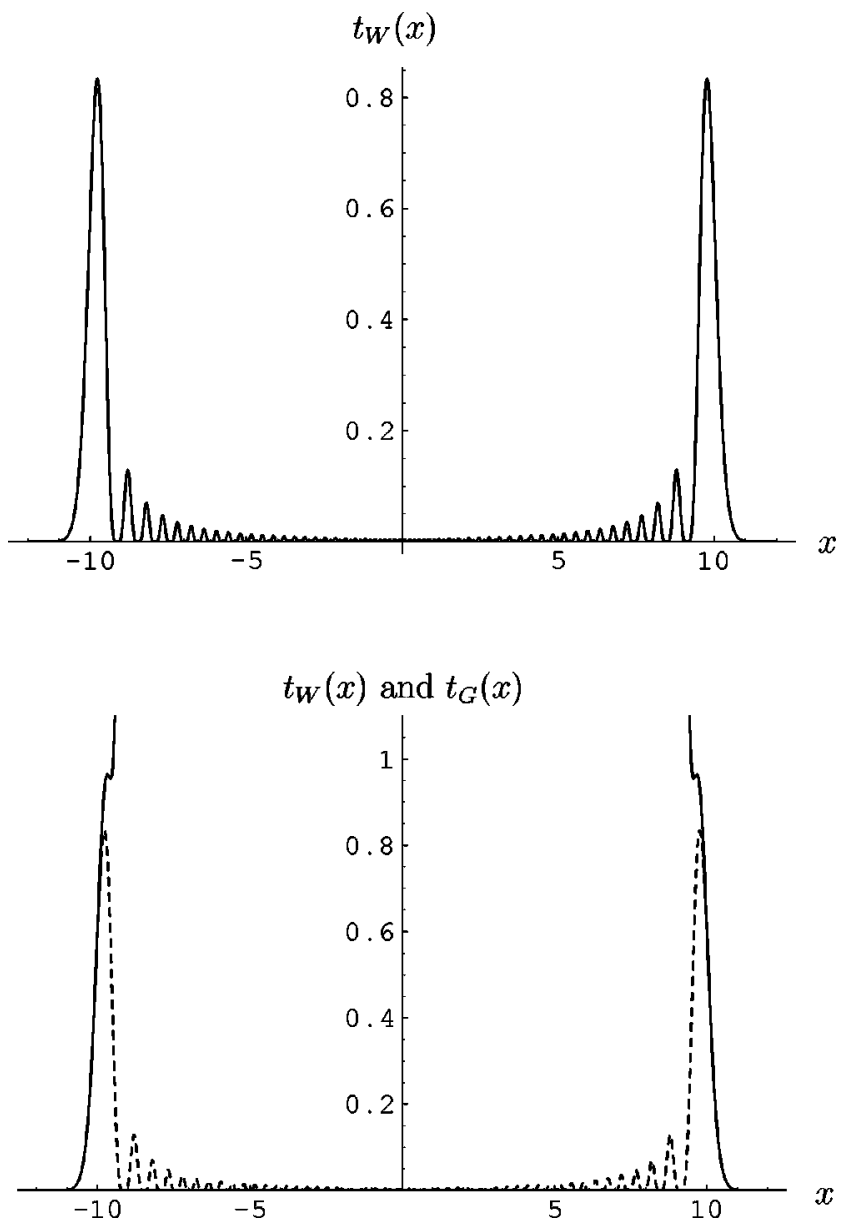

FIG. 2. Upper part of the figure shows the von Weizsäcker inhomogeneity kinetic energy $t_{W}(x)$ defined in Eq. (1), for $N=50$. Lower part shows the outer region of $t_{G}(x)$ plotted in full in the middle part of Fig. 1. The point to be emphasized is that $t_{G}$ (solid curve) rapidly tends to the von Weizsäcker kinetic energy $t_{W}(x)$ in the region where there is tunneling through the harmonic potential barrier.

We now turn to the region of $t_{G}(x)$, which reflects tunneling through the harmonic potential barrier. Figure 2 shows a plot of $t_{W}(x)$, the von Weizsäcker inhomogeneity kinetic energy in Eq. (1), in the upper part of the figure. The amplitude of $t_{W}$ is seen to be quite small inside the classically allowed region. The lower part of Fig. 2 compares $t_{W}$ with the tailing of $t_{G}$ already shown in Fig. 1. There is quantitative accord very quickly between $t_{G}$ and $t_{W}$ in the tails of the $t_{G}$ curve. Thus, near the classically forbidden region, $t_{G}$ rapidly tends to $t_{W}$ given by Eq. (1) and, although we shall not reproduce the results here, we have found almost identical behavior in the tail of $t_{G}$ for smaller values of $N$, namely, 10 and 20

Turning, more briefly, to the particle density $n(x)$, Fig. 3 shows the complete curve for $N=50$ calculated directly from Eq. (3). This Eq. (3) becomes less useful except nearer the tunneling region for $N=80$ and 100 and we have therefore reproduced only the outer part of $n(x)$ for these values. Comparing the complete curve of $n(x)$ for $N=50$ in Fig. 3 with $t(x)$ in the upper part of Fig. 1, it can soon be verified 


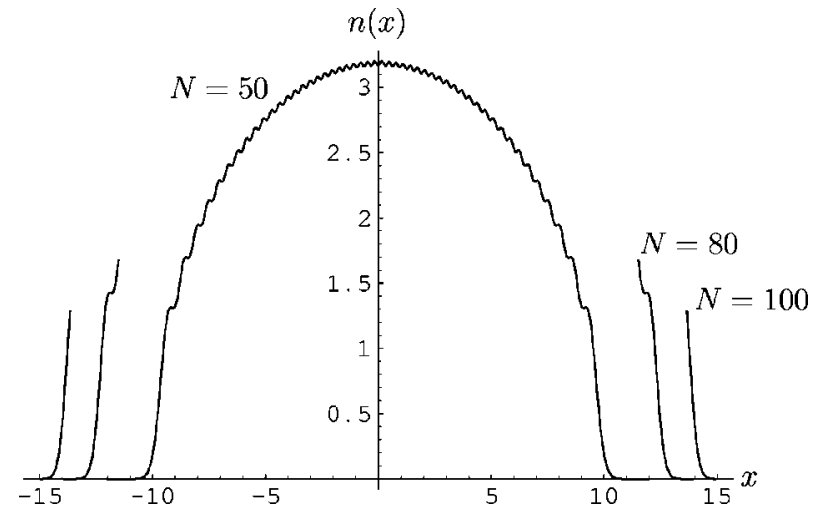

FIG. 3. Particle density $n(x)$ as calculated from Eq. (3) for $N$ $=50$. For $N=80$ and 100, direct calculation from Eq. (3) is most useful for giving the outer parts shown of density $n(x)$.

(as indeed from the curves in Ref. [1]) that the turning points of both $n(x)$ and $t(x)$ occur at the same values of $x$. This is immediately clear by referring to the exact analytical relation, now involving the first derivative $t^{\prime}(x)$ of $t(x)$ :

$$
\frac{t^{\prime}(x)}{n^{\prime}(x)}=N-\frac{1}{2} x^{2}
$$

which can be obtained by straightforward manipulation from Ref. [3]. It is, to us, remarkable that, on dividing curves obtained by differentiating both $t(x)$ in Fig. 1 and $n(x)$ for $N=50$ in Fig. 3, the quotient of two such highly structured curves should have the entirely smooth parabolic form of the RHS of Eq. (10).

Of course, we must stress that the Green's operator approach of Vignolo et al. [1] comes into its own for $N$ =1000: one needs only to modify the tail of their curve to avoid the (tiny) negative region by using formula (9) to correct $t(x)$ by $n^{\prime \prime}(x) / 4$. However, in the tails, $t_{G} \rightarrow t_{W}$ as emphasized in Fig. 2 for smaller $N$ values of 50 and 100.

We conclude by noting that after submission of the work of Vignolo et al. [1], the kinetic-energy functional they refer to in the penultimate paragraph of their Letter was obtained exactly [5]. It takes the form

$$
T[n]=T_{W}[n]+\int_{-\infty}^{\infty} t_{T F}[n] \xi[n] d x,
$$

where

$$
\xi[n] \equiv \alpha_{N}+\frac{1}{2} \int_{0}^{x} d s \frac{\left[n^{\prime}(s)\right]^{3}}{n^{2}(s) t_{T F}(s)} .
$$

Here $\alpha_{N}=\left[t(0)-t_{W}(0)\right] / t_{T F}(0)$ is

$$
\alpha_{N}= \begin{cases}\frac{2^{2 N-2}(2 N+1) \Gamma^{4}[(N+1) / 2]}{\pi \Gamma^{2}(N)}, & N \text { odd }, \\ \frac{2^{2 N-4}(2 N-1) \Gamma^{4}(N / 2)}{\pi \Gamma^{2}(N)}, & N \text { even, }\end{cases}
$$

while the Thomas-Fermi kinetic energy density $t_{T F}[n]$ is given by

$$
t_{T F}[n]=\frac{\pi^{2}}{6} n^{3}
$$

From Eq. (13) we can see that the parameter $\alpha_{N}$ goes very rapidly to unity as $N$ grows, and even for small $N$ the deviation from unity is slight.

As an illustration of Eq. (12) we have plotted from the results of the present study the function $\xi(x)$ versus $x$ in the upper part of Fig. 4 for the cases when $N=20$ and 50. Then, in the lower part of Fig. $4 \xi(x) t_{T F}(x)$, modified by $n^{\prime \prime}(x) / 4$, is plotted for the same $N$ values. The result is a short-range function, reflecting directly that $t_{G} \rightarrow t_{W}$ near the classically forbidden region.

In summary, the present study, being essentially analytical in character, complements the numerical investigation of Vignolo et al. [1] on noninteracting fermions with harmonic
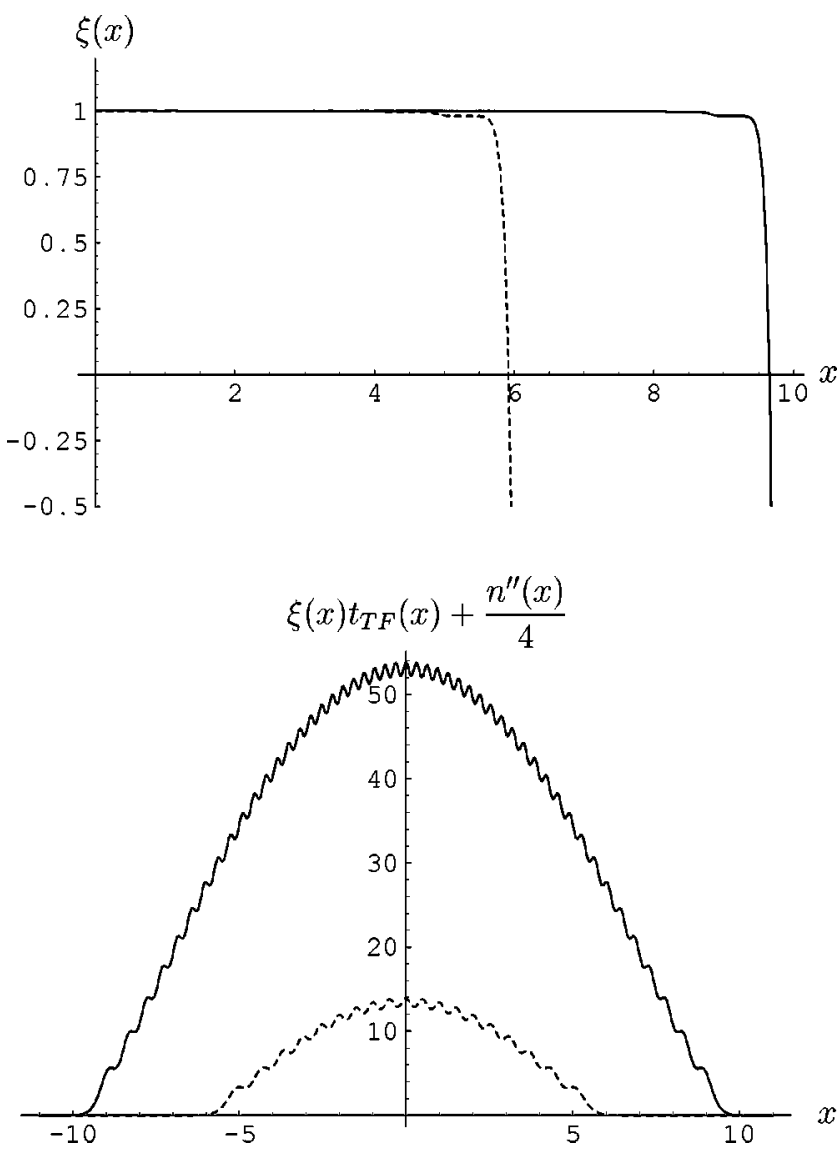

FIG. 4. Illustration of kinetic-energy density functionals in Eqs. (11) and (12). Upper part: plot of $\xi$ versus $x$ as defined in Eq. (12) for $N=20$ and 50 (solid). Note for comparison that, for $N=1$, $\xi(x)=(6 / \pi)\left((1 / 2)-x^{2}\right) \exp \left(2 x^{2}\right)$ and therefore has nodes at $x=$ $\pm \sqrt{2} / 2$, becoming negatively infinite for large $x^{2}$. It is to be stressed that $\xi(x) t_{T F}(x)$, for $N=1$ also, equals $(1 / \sqrt{\pi})(1 / 2$ $\left.-x^{2}\right) \exp \left(-x^{2}\right)$ and integrates to make zero contribution to the total kinetic energy in Eq. (11): i.e., $T=T_{W}$ for $N=1$, which is evidently correct. Lower part: $\xi(x) t_{T F}(x)+n^{\prime \prime}(x) / 4$ for the same values of $N$. 
confinement in one dimension using the Green's operator technique. Their numerical method comes into its own forlarge $N$ (1000 in their work), when the inset to their Fig. 4, which is in the present notation $2 t(x)$ for $N=1000$, is modified in a minor way for the difference between $t$ and $t_{G}$ in Eq. (9). The use of $t_{G}(x)$ is advocated here as (a) it is everywhere positive from its definition and (b) it rapidly approaches $t_{W}(x)$ in the tunneling regime.
One of us (N.H.M.) thanks Professor A. Holas for very valuable discussions embracing the present area at an earlier stage. This work was supported by the Spanish DGES (Grant No. PB98-0370) and Junta de Castilla y León (Grant No. CO2/199). Finally, it is a pleasure for N.H.M. to thank Professor J. A. Alonso for most generous hospitality during a visit to the University of Valladolid, which made possible this collaborative study.
[1] P. Vignolo, A. Minguzzi, and M.P. Tosi, Phys. Rev. Lett. 85, 2850 (2000).

[2] K. Husimi, Proc. Phys. Math. Soc. Jpn. 22, 264 (1940).

[3] G.P. Lawes and N.H. March, J. Chem. Phys. 71, 1007 (1979).
[4] A. Holas and N.H. March, Philos. Mag. B 69, 787 (1994).

[5] N.H. March, P. Senet, and V.E. Van Doren, Phys. Lett. A 270, 88 (2000). 